\title{
Line Separation from Topographic Maps Using Regional Color and Spatial Information
}

\author{
Pengfei Xu ${ }^{1}$, Qiguang Miao ${ }^{2}$, Tiange Liu ${ }^{3 *}$, Xiaojiang Chen ${ }^{1}$ and Dingyi Fang ${ }^{1}$ \\ ${ }^{1}$ School of Information Science and Technology, Northwest University, China \\ ${ }^{2}$ School of Computer Science and Technology, Xidian University, China \\ ${ }^{3}$ School of information science and engineering, Yanshan University, China \\ pfxu@nwu.edu.cn,qgmiao@xidian.edu.cn, liutiange@ysu.edu.cn, \\ xjchen@nwu.edu.cn,dyf@nwu.edu.cn
}

\begin{abstract}
The lines in topographic maps are difficult to be separated from each other because of their confusing colors. To solve this problem, we propose a novel line separation method using their regional color and spatial information. Firstly, we divide the lines into lots of circular regions with a certain diameter, and consider these regions as the basic processing units. Then based on a new concept of regional color confusion, we classify all the divided circular regions into two kinds of regions by whether the color is pure or mixed. Further, for pure color regions, a fuzzy clustering algorithm with Gaussian kernel can be used to cluster them into different lines based on their color information. Meanwhile, we determine the memberships of the mixed color regions according to their spatial relations with the clustered pure color regions. The concept of regional color confusion is proposed to reduce the influences of the confusing colors to line separation, and the spatial relations are utilized to solve the problems of the membership determination of the mixed color regions. The experimental results demonstrate that our method can achieve higher accuracy compare with other two state-of-the-art methods, which provides a novel idea for line element segmentation from scanned topographic maps.
\end{abstract}

\section{Introduction}

Topographic map carries a wealth of information of this planet over a very long period[Chiang et al., 2014]. There are a large number of historical topographic maps, which have been created in the past several centuries, and most of those maps were scanned and stored as raster images ${ }^{1,2,3,4}$.

\footnotetext{
* Corresponding author:liutiange@ysu.edu.cn

${ }^{1}$ https://www.old-maps.co.uk//

${ }^{2} \mathrm{http}: / /$ historicalmaps.arcgis.com/usgs/

${ }^{3}$ https://www.davidrumsey.com/

${ }^{4} \mathrm{http}: / / \mathrm{www}$. oldmapsonline.org/
}

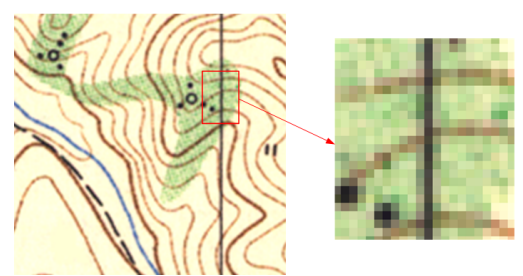

Figure 1: A map image with poor quality

The information contained in those digital maps including landscape ecology, land-cover changes and urbanization is very precious for many research topics, which leads to the automatic recognition of geographic elements in those topographic maps become essential. In topographic maps, a wide variety of geographical elements including contour lines, rivers, lakes, roads, and etc., are intertwined recorded [Chiang et al., 2011][Chen et al., 2006]. In order to efficiently and accurately recognize different geographic elements, element separation should be performed in the first place [Chiang et al., 2014]. However, color distortion happens due to the oxidation and pollution, during the long-term storage. Besides, false color caused by RGB channels misalignment and color aliasing induced by the scanner's point spread function also frequently happen in the scanning process. All these facts result in the poor quality of obtained map images (As shown in Figure 1). So our main challenge is how to obtain satisfied element separation results for these poor map images. In topographic maps, line elements carry most geographic information [Liu et al., 2016a][Miao et al., 2013]. Thus, in this paper we focus on segmenting lines including all the straight lines and other curve lines.

At present, most of element separation methods for the topographic maps regard maps as a set of pixels [Khotanzad and Zink, 2003][Chang et al., 2014a], and utilize the color of pixels and very limited spatial information to classify each pixel into different layers. These pixel-based methods rarely consider the regional features of geographic elements, and thus hardly deal with the problems of color distortion, false color and color aliasing. The results of these methods usually show with "pepper and salt" noise and poor continuity of lines, as shown in Figure 2. 


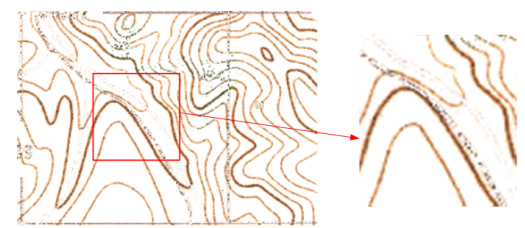

Figure 2: The contour line separation results with "salt and pepper" by a traditional method

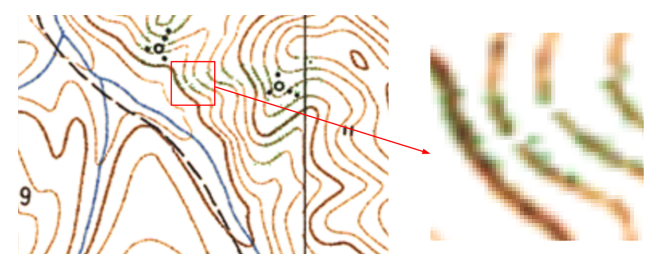

Figure 3: Lines with lots of colors in topographic maps

In 2010, Chen et al. proposed an object-oriented automatic segmentation algorithm [Chen et al., 2010], which considers line segments as the processing units for the first time. Compared with the traditional pixel-based algorithms, their results are significantly improved. However, the color information of each line segments is expressed by the average color of these lines, which would bring statistical mistakes when false color, color distortion, and even elements overlapping happened, as shown in Figure 3. In order to solve this problem, Liu et al. in 2016 [Liu et al., 2016a] defined the majority color of the lines as their main color, which is used to separate the lines. However, this kind of statistical method works well only for the long line segments with many pixels, but it is not applicable for the short lines with color distortion. Further, Liu et al. proposed another line separation algorithm based on region growing [Liu et al., 2016b], which has the idea of separating lines under the guidance of regional information. Nevertheless, this method does not make a special process for the regions with more chaotic colors (especially for node regions), which results in a number of fractures at the intersection points in the results.

According to human visual system, there are some obvious reasons for that people can quickly and accurately identify the lines representing different geographic elements[Thorpe et al., 1996][Isik et al., 2014][Chang et al., 2014b]. Firstly, human focus on not only the single pixel, but also the details of a local region. They also analyze and synthesize the interrelationships among the regions, rather than sticking to the isolated regions for identification. Moreover, the pixels and their neighboring ones affect each other, and constitute some regions with certain color and texture features, as shown in Figure 4. Therefore, how to make full use of the regional features and the relationships among these regions is the cue that motivates us to accomplish element separation, especially for line separation focused by us in this paper.

Based on all the analysis above, we propose a novel line separation method based on regional color and spatial information. This new method can be regarded as a line separation process with two paths, which are responsible for separating two types of regions respectively. In this method, we divide the lines extracted from topographic maps into small circu-

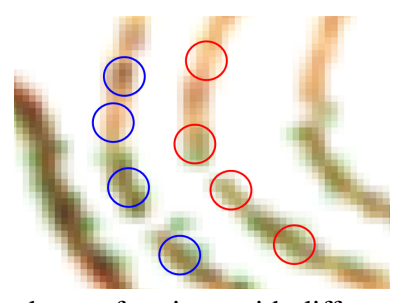

Figure 4: Lines made up of regions with different color and texture features

lar regions, which are classified into pure color regions and mixed color regions according to their color distribution. And then, a fuzzy clustering algorithm is used to separate pure color regions into different map layers, and the memberships of all the mixed color regions are determined based on their spatial relations with the separated pure color regions. Our method not only breaks through the limitation of considering the pixels as the basic processing units, but also overcomes the roughness of taking the whole line segments as the processing objects.

Our contributions can be summarized as follows:

1. We put forward the concept of regional color confusion, which is utilized to express the color distribution in a region. Based on this concept, the lines are classified into pure color regions and mixed color regions. Unlike the same processing strategy used for all the pixels or regions in previous existing methods.

2. We propose a new strategy for line separation from topographic maps: we put forward the mixed color regions, and we separate these special regions based on their spatial relationships between them and their neighbor pure color regions. Unlike the color information used for all the regions in these existing methods.

The remainder of this paper is organized as follows. In section 2, we review the related works. In Section 3, we analyze the color characteristics of different regions on the lines extracted from topographic maps and describe the proposed method in detail. The experimental results and analysis are given in Section 4 before concluding in Section 5.

\section{Related Work}

The geographic element separation from topographic maps has recently received much more attention [Chen et al., 2006][Chiang et al., 2009]. Various methods based on color histograms are applied to find an optimal threshold to segment topographic maps [Levachkine et al., 2001]. These methods have the advantages of lower computational complexity, and do not need any prior information. However, they are difficult to determine an optimal threshold, especially for the maps with poor quality. There also are several methods, which consider element separation as object classification [Mignotte, 2008], and in these methods, fuzzy classification techniques are more appropriate to solve the problem of the ambiguous color information in some topographic maps [Özdemir and Akarun, 2001][Özdemir and Akarun, 2002]. In 2015, Miao et al. proposed a fast image segmentation algorithm based on random probability sampling to solve the 


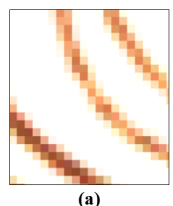

(a)

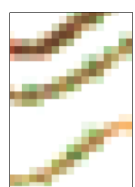

(b)

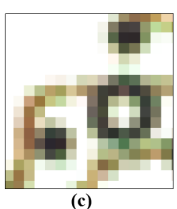

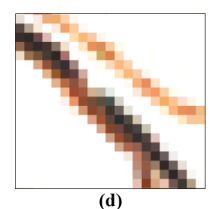

(d)
Figure 5: Four different types of regions on the lines extracted from topographic maps. (a)Contour lines with pure color; (b)Contour lines contaminated by vegetation; (c)Contour lines contaminated by background; (d)Contour lines contaminated by road lines.

problem that the existing methods have high computational complexity for the large maps, and achieved good performances [Miao et al., 2015].

However, the ambiguous color information of the pixels is always the bottleneck in the process of element separation. In order to solve this problem, Chen proposed an object-oriented algorithm for topographic map segmentation [Chen et al., 2010]. This algorithm takes independent lines as the processing units, which breaks through the limitation of using single pixel as the basic processing unit in the traditional pixel-based methods. Based on this idea, in 2016, Liu et al. proposed an algorithm based on the major color features of the lines [Liu et al., 2016a]. In his algorithm, he takes the main color of the lines for line separation instead of their average color. Moreover, by combining the color and spatial information of the lines. Liu proposed another contour-line color layer separation algorithm based on fuzzy clustering and region growing in 2016 [Liu et al., 2016b]. However, these methods still have difficulty in dealing with line separation in the complex topographic maps. For instance, they have no strategies for the determination of the memberships of the node regions. In this paper, we propose a novel line separation method based on regional color and spatial information to deal with some special regions better.

\section{Line Separation Using Regional Color and Spatial Information}

In general, there are four different types of regions on the lines extracted from topographic maps: the correct pure color regions (Figure 5 (a)), the false pure color regions contaminated by other elements (Figure 5 (b)), the mixed color regions contaminated by background (Figure 5 (c)), and those contaminated by other lines (Figure $5(\mathrm{~d})$ ).

In order to separate all these four different types of regions into their own map layers, we design a new line separation method using regional color and spatial information, and the framework of the proposed method is shown in Figure 6.

\subsection{The Region Division and Classification}

Before dividing regions, we extract all the lines from the original topographic maps using the linear element extraction algorithm proposed by Miao [Miao et al., 2013]. Then all the extracted lines are divided into a number of consecutive circular regions by taking the pixels on their skeleton lines (which is obtained by morphological thinning algorithm [Gonzalez et al., 2014], as shown in Figure 7) as the center points and their average width as the diameter. As shown in Figure 8 (a),

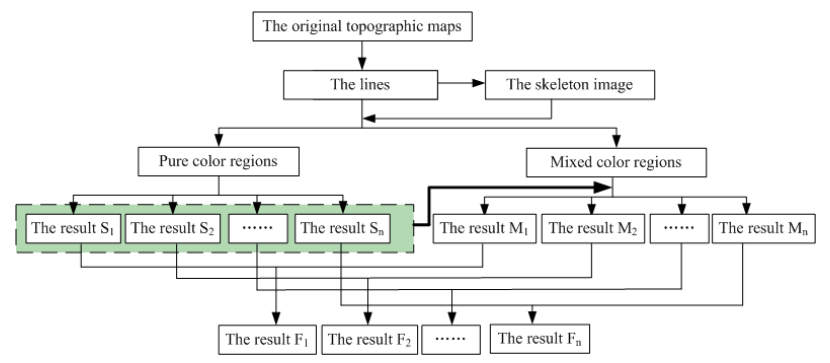

Figure 6: The framework of the proposed method

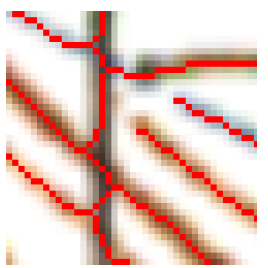

Figure 7: The skeleton lines

assuming that there are $m$ pixels on the skeleton line $L$, then a circular region $R$ on $L$ can be obtained by taking a certain pixel $l_{j}\left(x_{j}, y_{j}\right)(j=1,2, \ldots, m)$ on $L$ as the center point and the width $d$ of $L$ as the diameter. Further, all pixels in this circular region are grouped together as the minimum processing unit for line separation later. Since the skeleton lines basically retain the original shapes of the lines, it is possible for us to trace these lines from $l_{1}\left(x_{1}, y_{1}\right)$ to obtain a series of circular regions along the skeleton lines, which can be seen in Figure 8 (b).

Furthermore, we put forward the concept of regional color confusion to represent the complexity of color distribution in a certain region, and it can be expressed by formula (1).

$$
E_{R}=-\sum_{j=1}^{c} P\left(h_{j}\right) \log _{2} P\left(h_{j}\right)
$$

where $E_{R}$ is the value of regional color confusion within the region $R, P\left(h_{j}\right)$ is the proportion of the pixels with the color $h_{j}$ in region $R$, and $c$ is the total number of colors in $R$.

Compared with the mixed color region, a pure color region has much less complexity of color distribution, therefore, $E_{R}$ can be utilized to determine whether a circular region is a pure color region or a mixed color region, and which can be expressed by formula (2).

$$
\begin{cases}E_{R} \leq T & R \text { is a pure color region } \\ E_{R}>T & R \text { is a mixed color region }\end{cases}
$$

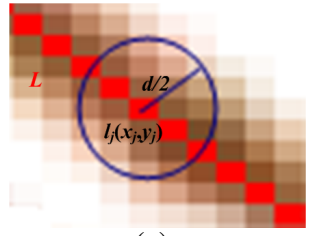

(a)

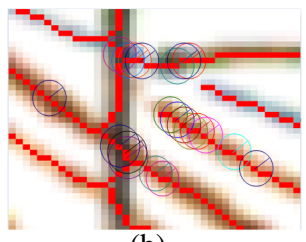

(b)
Figure 8: Lines and their circular regions. (a)A circular region $R$; (b)Lots of regions. 


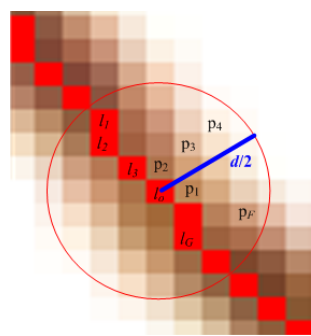

Figure 9: The way to obtain the color features of a pure color region.

where the value of $\mathrm{T}$ is about 4.0 , which is an experimental value obtained from lots of these two types of regions.

\subsection{Region Separation for The Pure Color Regions}

The color information of the whole line is affected by all its pixels, but they have varying degrees of influence to the line's color. Normally, the pixels far from the skeleton line have lower ability to represent the true color of this line than those near to the skeleton line, since they are easily influenced by background. Therefore, we calculate the regional color features based on the spatial relationships of each pixel from the central pixels.

It is assumed that there is a pure color region with a pixel $l_{O}\left(x_{O}, y_{O}\right)$ on its skeleton line as the center point, and the line width $d$ as the diameter, as shown in Figure 9. This region has $m_{o}$ skeleton pixels $l_{G}, G \in\left\{1,2, \ldots, m_{O}\right\}$, and $n_{o}$ nonskeleton pixels $p_{F}, F \in\left\{1,2, \ldots, n_{O}\right\}$.

For a non-skeleton pixel $p_{F}$, its corresponding weight $\omega_{F l}$, which can express its influence to the line's color, is calculated by formula (3),

$$
\omega_{F l}=\frac{1}{D_{F l}}
$$

where $D_{F l}$ is the minimum Euclidean distance from the nonskeleton pixels $p_{F}$ to the skeleton line $l_{G}$.

$$
\begin{aligned}
& D_{F l}=\min \left(\left\|p_{F}-l_{G}\right\|\right) \\
& \quad\left(F \in\left\{1,2, \ldots, n_{O}\right\} ; G=1,2, \ldots m_{O}\right)
\end{aligned}
$$

Then the color features of the pure color region $C_{R}$ can be calculated by formula (5):

$$
C_{R}=\left(\sum_{F=1}^{n_{O}}\left(\frac{\omega_{F l}}{\sum_{F=1}^{n_{O}} \omega_{F l}} \times C_{F}\right)+\frac{\sum_{G=1}^{m_{O}} C_{G}}{m_{O}}\right)
$$

where $C_{F}$ is the color information of the non-skeleton pixel $p_{F}$, and $C_{G}$ is the color information of the skeleton pixel $l_{G}$. It should be noted that all the color features are calculated on CIE L*a*b* color space [Liu et al., 2016b].

When the color features of all the pure color regions are obtained, a fuzzy clustering algorithm is used to separate these regions [Miao et al., 2015].

\subsection{Region Separation for The Mixed Color Regions}

Unlike region separation for the pure color regions, the mixed color regions, of which their centers connect to each other

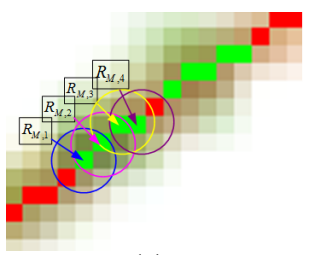

(a)

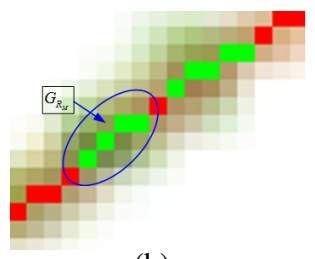

(b)
Figure 10: The schematics of the mixed color regions and their groups. (a)The mixed color regions; (b)The group of the mixed color regions.

in the skeleton line, are grouped together as a region group $G_{R_{M}}$, as shown in Figure 10. Subsequently, these groups are classified based on their spatial relationships between them and their neighbor pure color regions which have been separated before.

Since a mixed color region may be composed of several lines, it is allowed to belong to more map layers, which can accurately show the true attributes of each mixed color region. In topographic maps, there are four cases of the spatial relations between the groups of the mixed color regions and their neighbor pure color regions, so we can determine the memberships of these groups by performing the following procedures.

1) For the isolated groups, there are no neighboring pure color regions, as shown in Figure 11 (a), then $G_{R_{M}} \in \Phi$, and their memberships need to determine manually. However, this situation, which shows the overlap between two lines with the same shape from the top to end, is rarely appeared in topographic maps.

2) When there is a line segment with parts of the pure color regions labeled $L_{1}$, and end with a group of the mixed color regions, as shown in Figure 11 (b), then $G_{R_{M}} \in L_{1}$.

3 ) If there is one group of the mixed color regions connected between two line segments of the pure color regions with the same label $L_{1}$, as shown in Figure 11 (c), then $G_{R_{M}} \in$ $L_{1}$.

4) In a more complex case, if one group of the mixed color regions connects two or even more line segments of the pure color regions with different labels, which may be $L_{1}, L_{2}, \ldots, L_{Q}$, as shown in Figure11 (d), then $G_{R_{M}}\left\{\begin{array}{l}\in L_{1} \\ \in L_{2} \\ \ldots \\ \in L_{Q}\end{array}\right.$.

Up to now, we have accomplished the separation of all the pure color regions and the mixed color regions, and the final separation results can be obtained.

\subsection{Summary of The Proposed Method}

In summary, there are mainly five formulas in the proposed method which being successively performed to achieve the segmentation. Formula (1) is used to calculate the complexity of color distribution in a certain region of the lines. Formula (2) is utilized to determine whether a circular region is a pure color region or a mixed color region. Formula (3) and 


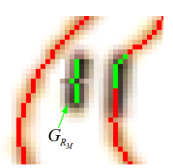

(a)

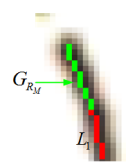

(b)

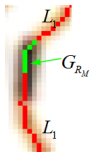

(c)

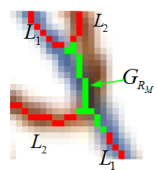

(d)
Figure 11: The separation of the groups of the mixed color regions.(a) $G_{R_{M}} \in \Phi$; (b) $G_{R_{M}} \in L_{1}$; (c) $G_{R_{M}} \in L_{1}$; (d) $G_{R_{M}} \in$ $\left\{L_{1}, L_{2}, \ldots, L_{Q}\right\}$

Formula (4) is combined to calculate the weights, which can express their confidence of representing the areas' colors. Finally, formula (5) is used to calculate the color features of the pure color region, which will be put into the subsequent clustering algorithm to separate these pure color regions. For further demonstrate the algorithm, the pseudo code is listed in the following.

\section{Algorithm 1 : Line separation using regional color and spatial information}

Input:Input image I (an original topographic map)

The total number $C$ of colors in image $I$.

Output:The separated maps: $M_{1}, M_{2}, \ldots, M_{C}$

1. Apply line extraction algorithm to obtain the linear element image $I_{\text {line }}$

2. Divide lines of $I_{\text {line }}$ into $n$ small regions $R_{i}, i=$ $1,2, \ldots, n$

3. Classify $R_{i}$ into $p$ pure color regions $R_{l}^{\text {pure }}, l=$ $1,2, \ldots, p$, and $q$ mix color regions $R_{m}^{m i x}, m=1,2, \ldots, q$

4. For $R_{l}^{\text {pure }}$, apply Fuzzy clustering method to separate $R_{l}^{\text {pure }}$ into $C$ classes $M_{c}, c=1,2, \ldots, C$

5. For $R_{m}^{m i x}$, calculate the relationship between $R_{m}^{m i x}$ and all $M_{c}$, and merge $R_{m}^{m i x}$ into the corresponding $M_{c}$

6. Obtain the separated maps: $M_{1}, M_{2}, \ldots, M_{C}$

\section{Experiments and Analysis}

In order to verify the effectiveness and feasibility of the proposed method, three original topographic maps with different sizes are used as testing images, as shown in Figure 12. Besides, FCM algorithm using a single pixel as the basic processing unit [Miao et al., 2015] and Liu's method [Liu et al., 2016a] are exploited as the comparison methods. Moreover, we make subjective and objective evaluations on the experimental results respectively. Since the contour lines are the most important geographical elements in geographic information systems, the brown maps of contour lines are given for Map B and Map C with large sizes, while the other separation maps are not shown because of space limitation of this paper.

From the selected experimental results which are shown in Figure 13-17, we can see that FCM algorithm has unsatisfied performances for these maps: (1) There are lots of noise in the result maps due to the complex color distribution in topographic maps, as shown in Figure 14(b), Figure 16 (b) and Figure 17(b). (2) Many gaps appear on the segmented

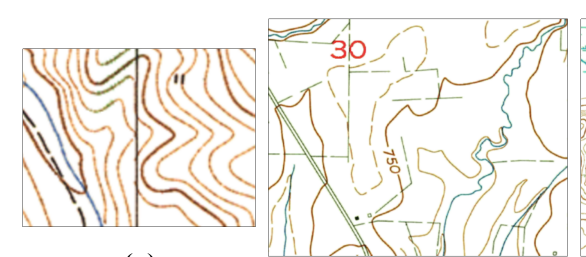

(b)

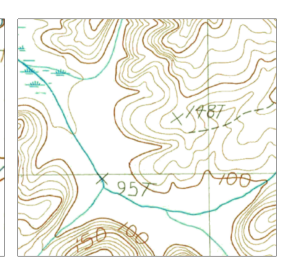

(c)
Figure 12: The testing topographic maps. (a)Map A; (b)Map B; (c)Map C.

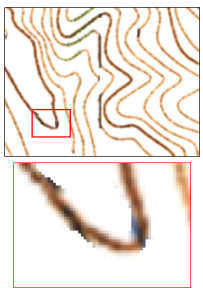

(a)

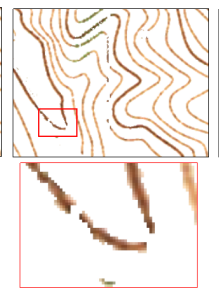

(b)

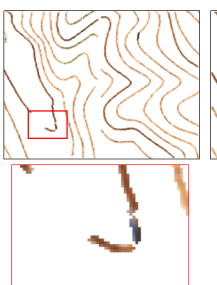

(c)

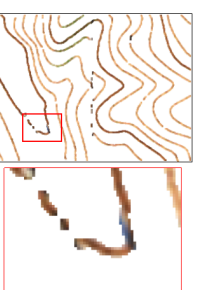

(d)
Figure 13: The brown results obtained from Map A with zoomedin patches. (a)The ground truth; (b)FCM; (c)Liu's method; (d)our method.

lines. The performance of Liu's method has a great improvement. There is much less noise in the results, and the lines have better continuity as well. However, there is still some inaccurate separation in some places, especially for the lines with many nodes, which results in the gaps appear around the nodes, as shown in Figure 15 (c), Figure 16 (c), and Figure 17(c). Compared with these two methods, our method can achieve better performances. We use two types of regions as the basic processing units instead of pixels, which leads to less noises in the results. Besides, the spatial information is utilized to determine the memberships of the mixed color regions, and some mixed color regions may have more than one memberships, which not only can improve the separation accuracy, but also handle the nodes to get much better continuity of the result lines, as shown in Figure 13 (d), Figure16 (d), and Figure 17 (d).

Furthermore, we use the rate of correct separated pixels (RCP), the rate of false separated pixels (RFP) [Miao et al., 2015] and the rate of connected components (RCC) to evaluate the result maps.

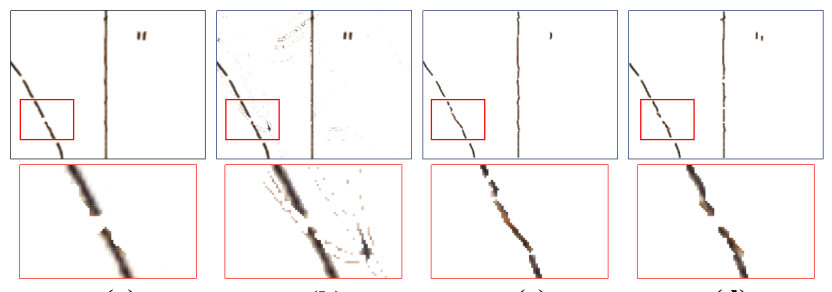

(a)

(b)

(c)

(d)

Figure 14: The black results obtained from Map A with zoomedin patches. (a)The ground truth; (b)FCM; (c)Liu's method; (d)our method. 


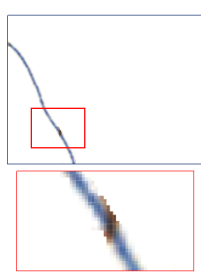

(a)

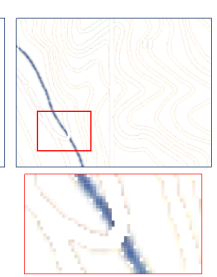

(b)

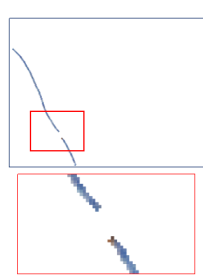

(c)

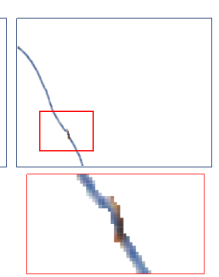

(d)
Figure 15: The blue results obtained from Map A with zoomedin patches. (a)The ground truth; (b)FCM; (c)Liu's method; (d)our method.

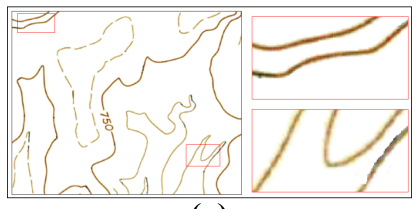

(a)

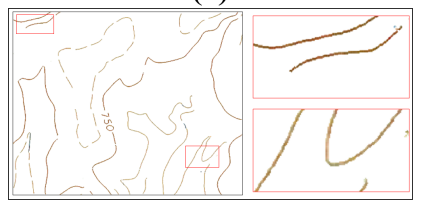

(c)

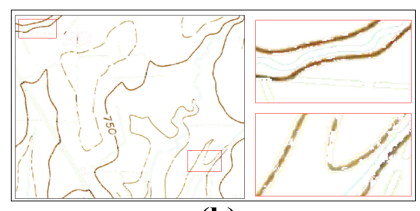

(b)

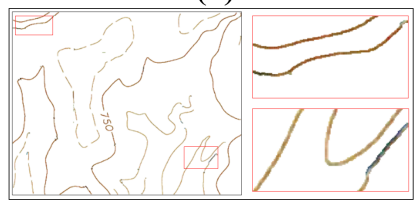

(d)
Figure 16: The brown maps obtained from Map B with zoomedin patches. (a)The ground truth; (b)FCM; (c)Liu's method; (d)our method.

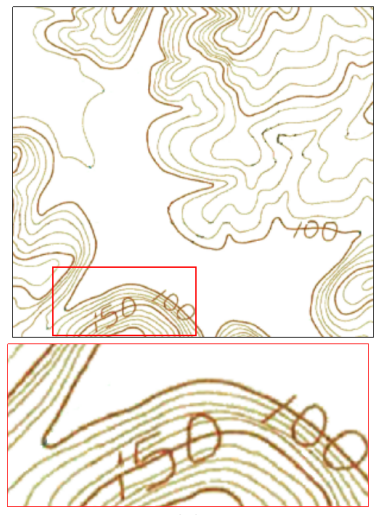

(a)

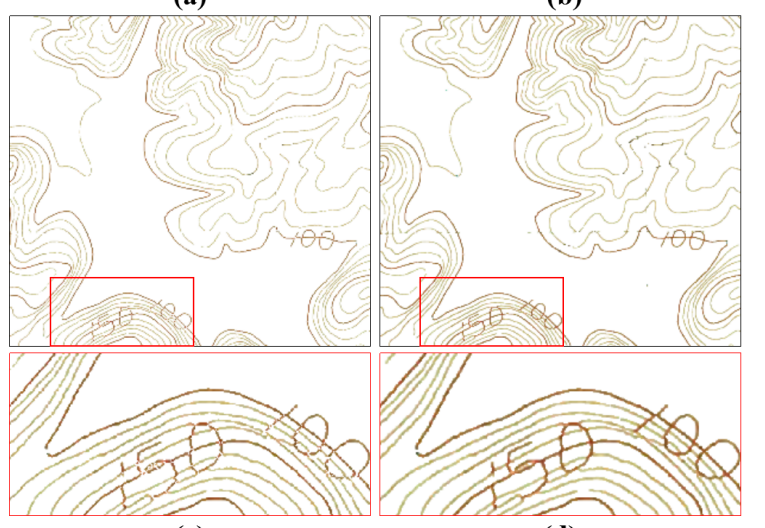

(c)

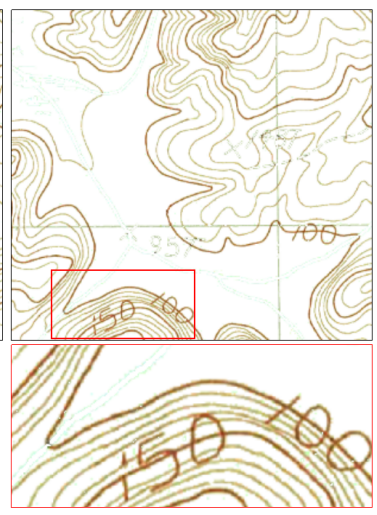

(b)

(d)
Figure 17: The brown maps obtained from Map C with zoomedin patches. (a)The ground truth; (b)FCM; (c)Liu's method; (d)our method.

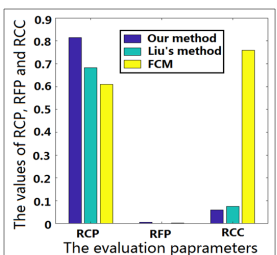

(a)

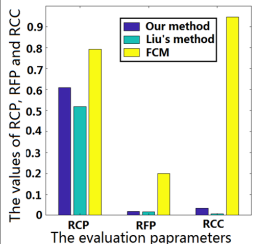

(b)

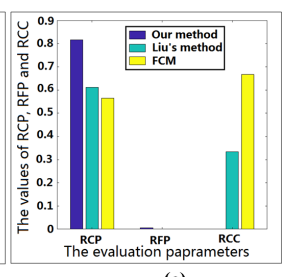

(c)
Figure 18: The evaluations of the results by different methods on Map A. (a)Brown map; (b)Black map; (c)Blue map.

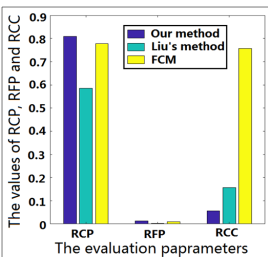

(a)

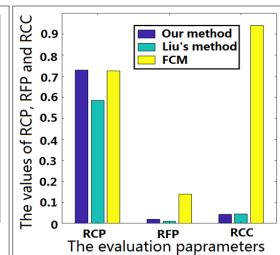

(b)

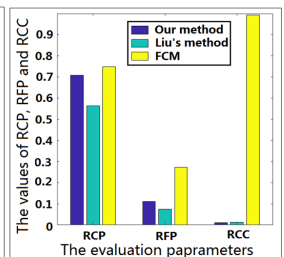

(c)
Figure 19: The evaluations of the results by different methods on Map B. (a)Brown map; (b)Black map; (c)Blue map.

The rate of the connected components:

$$
R C C=\frac{L N-T L N}{\max \left\{L N_{1}, L N_{2}, \ldots, L N_{m}\right\}}
$$

where $L N$ is the number of the connected components in the result maps, $T L N$ indicates the number of the connected components in their corresponding ground truth, and $\max \left\{L N_{1}, L N_{2}, \ldots, L N_{m}\right\}$ is the maximum value of $L N$ in the result maps obtained by $m$ kinds of methods.

In the following, we evaluate all the results with the above metrics, and the corresponding evaluations are shown in Figure 18 to Figure 20. It can be seen that FCM has lower RCP in most result maps, and has much higher RFP and much worse RCC in all the results. The results obtained by Liu's method have obvious improvement, the lines in the result maps are more continuous, and fewer false separated pixels appear. So his method works better in RCC and RFP than FCM. In contrast to these two methods, our method has the best performances. The lowest RCC shows its separated lines has the most continuity, and its higher RCP and lower RFP show that it can separate more pixels into their own maps correctly.

\section{Conclusion}

There are many challenges for line separation from topographic map with low quality. This paper proposes a new

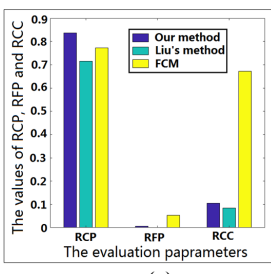

(a)

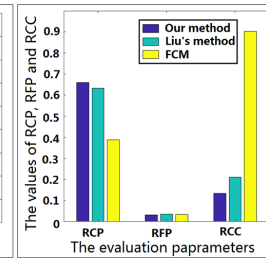

(b)

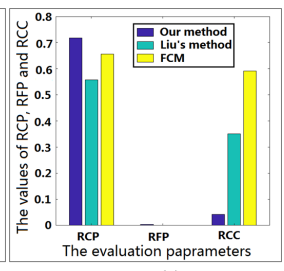

(c)
Figure 20: The evaluations of the results by different methods on Map C. (a)Brown map; (b)Black map; (c)Blue map. 
line separation method by utilizing regional color and spatial information. It divides the lines into two types of regions, which are then separated to their own result maps respectively. Especially for the mixed color regions, the spatial relations between them and the pure color regions are used to determine their memberships, which overcomes the problem of the false separation of the contaminated pixels by background or other elements, and he experimental results show that our method has a better performance.

\section{Acknowledgments}

The work was jointly supported by the National Natural Science Foundations of China under grant No. 61502387, 61702415, 61772396, 61472302; Natural Science Foundation of Shaanxi Province, under grant No.2016JQ6029, 2017JQ4003; The 59th China's Post-doctoral Science Fund No. 2016M592832. Natural Science Foundation of Hebei Province of China under grant No.F2018203096, the 61th China's Post-doctoral Science Fund No.2017M611188

\section{References}

[Chang et al., 2014a] Xiaojun Chang, Feiping Nie, Zhigang Ma, Yi Yang, and Xiaofang Zhou. A convex formulation for spectral shrunk clustering. pages 2532-2538, 2014.

[Chang et al., 2014b] Xiaojun Chang, Feiping Nie, and Sen Wang. Compound rank- $\mathrm{k}$ projections for bilinear analysis. IEEE Transactions on Neural Networks Learning Systems, 27(7):1502-1513, 2014.

[Chen et al., 2006] Yang Chen, Runsheng Wang, and Jing Qian. Extracting contour lines from common-conditioned topographic maps. IEEE Transactions on Geoscience \& Remote Sensing, 44(4):1048-1057, 2006.

[Chen et al., 2010] Hong Chen, Xiaoan Tang, Chenhao Wang, and Zhe Gan. Object oriented segmentation of scanned topographical maps. Journal of Image \& Graphics, 15(9):1416-1424, 2010.

[Chiang et al., 2009] Yao Yi Chiang, Craig A. Knoblock, Cyrus Shahabi, and Ching Chien Chen. Automatic and accurate extraction of road intersections from raster maps. Geoinformatica, 13(2):121-157, 2009.

[Chiang et al., 2011] Yao Yi Chiang, Stefan Leyk, and Craig A. Knoblock. Efficient and robust graphics recognition from historical maps. In International Conference on Graphics Recognition: New Trends and Challenges, pages 25-35, 2011.

[Chiang et al., 2014] Yao Yi Chiang, Stefan Leyk, and Craig A. Knoblock. A survey of digital map processing techniques. Acm Computing Surveys, 47(1):1-44, 2014.

[Gonzalez et al., 2014] Rafael C. Gonzalez, Richard E. Woods, and Steven L. Eddins. Digital image processing. Publishing House of Electronics Industry, 2014.

[Isik et al., 2014] Leyla Isik, Ethan M. Meyers, Joel Z. Leibo, and Tomaso Poggio. The dynamics of invariant object recognition in the human visual system. Journal of Neurophysiology, 111(1):91, 2014.
[Khotanzad and Zink, 2003] Alireza Khotanzad and Edmund Zink. Contour line and geographic feature extraction from usgs color topographical paper maps. Pattern Analysis \& Machine Intelligence IEEE Transactions on, 25(1):18-31, 2003.

[Levachkine et al., 2001] Serguei Levachkine, Aurelio Velázquez, Victor Alexandrov, and Mikhail Kharinov. Semantic analysis and recognition of raster-scanned color cartographic images. In Graphics Recognition Algorithms and Applications, International Workshop, Grec 2001, Kingston, Ontario, Canada, September 7-8, 2001, Selected Papers, pages 178-189, 2001.

[Liu et al., 2016a] Tiange Liu, Qiguang Miao, Pengfei Xu, Jianfeng Song, and Yining Quan. Color topographical map segmentation algorithm based on linear element features. Multimedia Tools \& Applications, 75(10):54175438, 2016.

[Liu et al., 2016b] Tiange Liu, Qiguang Miao, Pengfei Xu, Yubing Tong, Jianfeng Song, Yun Yang, Yun Yang, and Xiaojie Zhai. A contour-line color layer separation algorithm based on fuzzy clustering and region growing. Computers \& Geosciences, 88(C):41-53, 2016.

[Miao et al., 2013] Qiguang Miao, Pengfei Xu, Tiange Liu, Yyun Yang, Junying Zhang, and Weisheng Li. Linear feature separation from topographic maps using energy density and the shear transform. IEEE Transactions on Image Processing A Publication of the IEEE Signal Processing Society, 22(4):1546-1556, 2013.

[Miao et al., 2015] Qiguang Miao, Pengfei Xu, Tiange Liu, Jianfeng Song, and Xiaojiang Chen. A novel fast image segmentation algorithm for large topographic maps. Neurocomputing, 168(C):808-822, 2015.

[Mignotte, 2008] Max Mignotte. Segmentation by fusion of histogram-based $k$-means clusters in different color spaces. IEEE Transactions on image processing, 17(5):780-787, 2008.

[Thorpe et al., 1996] Simon Thorpe, Denis Fize, and Catherine Marlot. Speed of processing in the human visual system. Nature, 381(6582):520-522, 1996.

[Özdemir and Akarun, 2001] Dogan Özdemir and Lale Akarun. Fuzzy algorithms for combined quantization and dithering. IEEE Transactions on Image Processing, 10(6):923-931, 2001.

[Özdemir and Akarun, 2002] Doğan Özdemir and Lale Akarun. A fuzzy algorithm for color quantization of images. Pattern Recognition, 35(8):1785-1791, 2002. 